\title{
Aroma molecules as dynamic volatile surfactants: functionality beyond the scent
}

\author{
Oxana A. Soboleva, ${ }^{\#}$ Pavel V. Protsenko, ${ }^{\#}$ Vadim V. Korolev, ${ }^{\#}$ Jekaterina Viktorova, ${ }^{\dagger \%}$ Alexey \\ Yakuschenko, ${ }^{\dagger}$ Ruth Kudla, ${ }^{\ddagger}$ Jochen S. Gutmann, ${ }^{\ddagger \&}$ and Larisa A. Tsarkova*\#‡ \\ ${ }^{\#}$ Chair of Colloid Chemistry, Faculty of Chemistry, Moscow State University 119991 Moscow, 1-3 Leninskiye Gory, \\ Russia \\ ${ }^{\dagger}$ Isitfresh GmbH, Technologiezentrum am Europaplatz, Dennewartstraße 25,52068 Aachen, Germany \\ ${ }^{\ddagger}$ Deutsches Textilforschungszentrum Nord-West gGmbH (DTNW) Adlerstraße 1, 47798 Krefeld, Germany \\ ${ }^{\&}$ Department of Chemistry \& CENIDE, University Duisburg-Essen, Universitätsstraße 5, 45141 Essen, Germany
}

\begin{abstract}
Understanding of non-equilibrium processes at dynamic interfaces is indispensable for advancing design and fabrication of solid state and soft materials. The research presented here unveils specific interfacial behavior of aroma molecules and justifies their usage as multifunctional volatile surfactants. As non-conventional volatile amphiphiles we study commercially available poorly water-soluble compounds from the classes of synthetic and essential flavor oils. Their distinctive feature is high dynamic interfacial activity, so that they decrease the surface tension of aqueous solutions on a time scale of milliseconds. Another potentially useful property of such amphiphiles is their volatility, so that they notably evaporate from interfaces on a time scale of seconds. This behavior allows for control of wetting and spreading processes. A revealed synergetic interfacial behavior of mixtures of conventional and volatile surfactants is attributed to a decrease of the adsorption barrier as a result of high statistical availability of new sites at the surface upon evaporation of the volatile component. Our results offer promising advantages in manufacturing technologies which involve newly creating interfaces, such as spraying, coating technologies, ink-jet printing, microfluidics, laundry, stabilization of emulsions in cosmetic and food industry, as well as in geosciences for controlling aerosols formation.
\end{abstract}

\section{Introduction}

Odor compounds are aliphatic or aromatic amphiphilic molecules with varied carbon backbones and diverse functional groups, including aldehydes, esters, ketones, alcohols, alkenes, carboxylic acids, amines. Essential oils are generated through different biosynthetic routes and extracted from vegetable raw materials. The fragrance and cosmetic industries promoted the development of chemical synthesis of perfume molecules, ${ }^{1}$ as well as activated research on the structure-odor relationship. Odortriggering activity is an important part of specific signaling pathways in olfactory system, an evolutionally evolved remarkable molecular sensing mechanism. ${ }^{2,3}$ Features of molecules which can act as odorant-triggers are typically considered in terms of molecular weight, geometry, chirality, flexibility (vibrational frequencies and rotation), as well as biochemical (enzymatic) influence. Applications of aroma molecules are currently related to their biological activities (odor, antioxidant, antimicrobial). However, rich and complex composition of these poorly water soluble volatile amphiphiles defines their specific physicochemical properties beyond the scent function.

In this paper we disclose functional properties of aroma molecules, which are of high interest for material and surface science and which are envisaged to notably expand the application fields of this class of amphiphilic compounds. One of the important parameters which define the formation, properties and functions of aqueous solutions, dispersions and mixtures is the surface/interfacial tension $\gamma$ which is typically assumed to be an equilibrium value. This thermodynamic parameter defines the energetic costs associated with the existence of a phase boundary, e.g. between liquid and air. A standard approach to facilitate the formation of new surfaces is to apply surfactants - amphiphilic molecules, which concentrate in a thin sub-interfacial region, and thus decrease the surface/interfacial energy (Figure 1a). However, the systems in which a fresh surface is created may not be in an adsorption equilibrium, and therefore exhibit a time-dependent (dynamic) surface tension $\gamma(\mathrm{t})$. $^{4,5}$

For solutions of amphiphilic molecules the $\gamma(\mathrm{t})$ dependence may span from microseconds to minutes (or even hours) ${ }^{5-7}$ (Figure $1 b$ ). In the simplest case the time effect is controlled by the rate of the surfactant diffusion $D$ to the surface according to the diffusion equation:

$\frac{\partial C}{\partial t}=D \frac{\partial^{2} c}{\partial x^{2}}$

where $c$ is bulk concentration of the surfactant and $x$ is a distance measured away from the surface. Diffusivity $D$ depends on the molecular weight and molecular structure of a molecule (Figure $1 b$ ), so that in practical research the choice of surfactants is often considered in terms of their dynamic interfacial activity.

"Dynamic" interfaces with non-equilibrium adsorption state are relevant to a wide range of processes and technologies in which "fresh" surfaces are created (Figure 1c). 


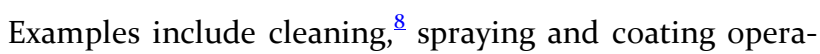
tions, i.e. liquids emerging from orifices or spray nozzles, 9 , 10 or bubbles and droplets formed within liquids, as in microfluidic devices. ${ }^{\underline{1}}$ In ink jet printing surface life-times of as low as fractions of a millisecond are of practical importance. ${ }^{12}$

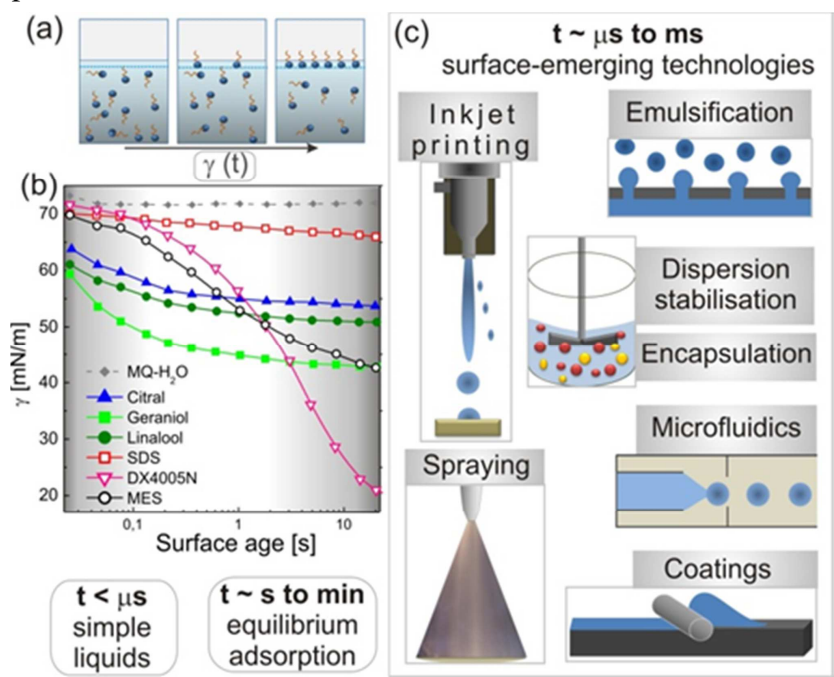

Figure 1(a) Schematic illustration of the time-dependent concentration growth in the sub-interfacial layer (dashed line) at liquid-air interface. (b) Examples of the kinetic curves of the surface tension of $\sim 0,3 \mathrm{~g} / \mathrm{l}$ solutions in MQ water of conventional surfactants (empty symbols) (Table S1, Supporting Information) and of volatile surfactants (filled symbols) (Table 1) measured with bubble pressure tensiometer at $25^{\circ} \mathrm{C}$ : dashed line - MQ water; red empty squares- sodium dodecyl sulfat (SDS); magenta down triangles$\mathrm{DX}_{4005} \mathrm{~N}$ fluorosurfactant; black circles - methylestersulfonat (MES); blue triangles - citral; green circles - linalool, orange squares - geraniol. (c) Examples of processes and technologies which involve formation of new surfaces at a timescale of milliseconds and below.

In this paper we introduce volatile surfactants (from a class of aroma molecules) which possess distinctive features such as volatility and a higher than conventional surfactants dynamic interfacial activity at a time scale of milliseconds (Figure $\mathrm{bb}$, Table 1 ). We note these unique and powerful features of aroma (fragrance) molecules remained until now unrevealed, so that intensive fundamental research on the effects of perfumes on the interfacial processes in complex colloidal systems has to be revisited. ${ }^{13}$ Here these properties are quantified using dynamic and static (equilibrium) surface tension measurements, and are further demonstrated in dynamic wetting experiments. We present a methodology allowing evaluating interactions of volatile surfactants with other component of complex liquids, and disclose a synergetic interfacial behavior of volatile and conventional surfactants, that offers breakthrough possibilities in surfacesemerging technologies. Preliminary conclusions are made with regards to the understanding the "structurefunction" relationship of volatile surfactants.

\section{Experimental Section}

Aroma compounds - flavor oils linalool, 1-decanol (both form Sigma-Aldrich), benzyl alcohol, citronellol and conventional surfactants SDS (Sigma-Aldrich), MES (PT Global Eco Chemicals), and DX4005N (Dynax) (Table $1 \mathrm{~S}$, Supporting information) have been used as received.

Solutions for the measurements of isotherms have been prepared by titration method via stepwise addition of weighted amounts of corresponding liquids (or stock solutions) to MQ water under stirring at constant temperature for 10 minutes at $25^{\circ} \mathrm{C}$, if not stated otherwise.

Solutions of conventional surfactants sodium dodecyl sulfat (SDS from Sigma-Aldrich), methylestersulfonat (MES from PT. Global Eco Chemicals Indonesia) anionic surfactants and $\mathrm{DX}_{400} \mathrm{~N}$ fluorosurfactant (Dynax Corporation) (Table $\mathrm{S} 1$, Supporting Information) have been prepared by dilution of stock solutions. The curves presented in Figure $1 b$ are exemplary measurements in course of titration experiments to obtain surface tension isotherm of respective surfactants.

Dynamic surface tension was measured by maximum bubble pressure method at $25 \pm 0.5^{\circ} \mathrm{C}$ using a SITA pro line t1oo apparatus (SITA GmbH, Germany) and capillary from polymethyl ether ketone (MEEK).

Measuring dynamic surface tension with the bubble pressure method enables high precision and flexibility without adjustment of the immersion depth. This is done by pumping air through a capillary into the liquid to be analyzed. The pressure within the bubble changes continuously as the radius growths. The surface tension is calculated from the deviation between pressure maximum and minimum. The calibration is carried out with MQ water. Thereby, the radius of the capillary $(0.8 \mathrm{~mm})$ is taken into account.

Static surface tension was measured by the pendant drop method at $25 \pm 1^{\circ} \mathrm{C}$ using horizontal microscope. The drop was continuously imaged via DCM-13o digital video camera. The value of the surface tension was determined from the shape analysis of the pendant drops by numerical integration of the Young-Laplace equation and approximation of the resulting droplet shape to experimentally obtained image. To determine the equilibrium surface tension, droplets of the surfactant solution were formed in a closed cuvette with an aliquot of a corresponding solution at the bottom of the cell to maintain the respective partial vapor pressure. To study the effect of the solution evaporation and the volatility of the surfactants on the surface tension, the drops have been formed in the open system (Figure 4a).

Spreading kinetics was studied in the experimental setup consisting of horizontal microscope equipped with a high-speed video camera (XIMEA xiQ), horizontal adjustable substrate support, and vertical dosing syringe with flat-tip needle mounted above the substrate. In a standard procedure a substrate is placed on substrate support, and a drop with a diameter of $\sim 1 \mathrm{~mm}$ is formed at the needle tip. The substrate support is gently raised until the drop contact with the substrate is initiated, and the drop is simultaneously transferred and spreads onto the substrate 
surface. Process of drop transfer and spreading is recorded with $432 \times 310$ pix resolution at a frame rate of $1000 \mathrm{fps}$. Geometrical parameters of the spreading drop (contact angle $\theta$ and drop base diameter $\mathrm{d}$ ) have been extracted from the sequence of the drop images using home-made software. For each system at least five drops have been reproduced. Freshly cleaned glass slides (cleaning procedure with chromic mixture followed by UV/ozone treatment) or Melinex ${ }^{\circledast}$ PCS peelable polymer slides have been used as substrates.

Jetting experiments have been done using pico-liter dosing system (PDDS module from Dataphysics, Germany). $30 \mathrm{pl}$ droplets of studied solutions have been ejected onto fresh polymer substrate (Milinex ${ }^{\circledast}$ PCS). The drop diameter and the contact angle have been monitored and analyzed using SCA Software.

\section{Results and Discussion}

The interfacial behavior of amphiphilic organic substances was studied in connection to their molecular structure and related physico-chemical parameters (Table 1). Figure 2a displays surface tension of solutions of three alcohols measured with maximum bubble pressure tensiometer which assesses the pressure development in a gas bubble (fresh interface) at a tip of a capillary immersed into a liquid (Inset in Figure 2b)..$^{4}$ This method allows a time window from milliseconds to hundreds of seconds over which dynamic surface tension measurements can be made. ${ }^{5}$ Figure 2 a compares surface tension isotherms of decanol, linalool and benzyl acetate in dynamic (at 40 $\mathrm{ms}$ ) and in quasi-static (at $20 \mathrm{~s}$ ) regimes. Examples of kinetic curves (Figure $2 \mathrm{~b}$ ) are selected for solutions which exhibit $\gamma$ of $\sim 57 \mathrm{mN} / \mathrm{m}$ at 20 s surface age (marked as blue area in Figure $2 \mathrm{a}$ ). The corresponding concentrations in bulk indicate the amount of a solute required to produce $\sim 15 \mathrm{mN} / \mathrm{m}$ reduction in the surface

(a)
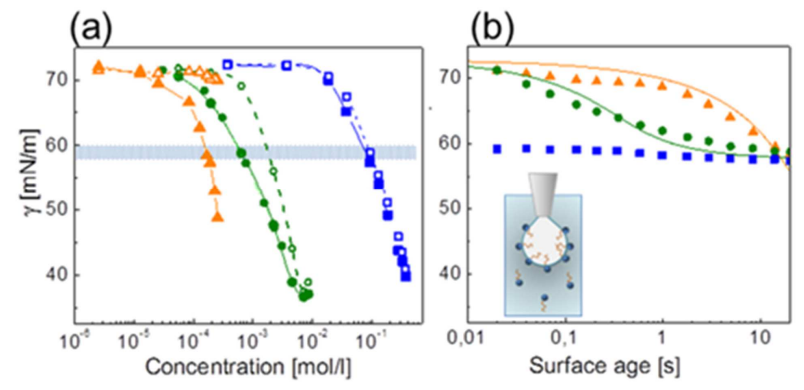

Figure 2. (a) Surface tension measured with the bubble pressure tensiometer at $45 \mathrm{~ms}$ (open symbols) and at $20 \mathrm{~s}$ (filled symbols) as a function of the molar concentration in MQ water (log scale) of benzyl alcohol (blue rhombs), linalool (green circles) and of decanol (orange triangles). (b) Surface tension versus surface age (of a bubble) for the selected solutions of decanol, linalool and benzylalchohol with concentrations of $0,03 \mathrm{~g} / \mathrm{L}(0,00019 \mathrm{~mol} / \mathrm{L}), 0,116 \mathrm{~g} / \mathrm{L}(0,0075 \mathrm{~mol} / \mathrm{L})$ and $10 \mathrm{~g} / \mathrm{l}(0,092 \mathrm{~mol} / \mathrm{L})$, respectively. The surface tension at $20 \mathrm{~s}$ surface age of each solution is $\sim 58 \pm 1 \mathrm{mN} / \mathrm{m}$. The solid lines are fits according to Ward and Tordai model (Equation 2, Table S2 and Figure S1, Supporting Information). Sketch illustrates the measurement cell in bubble pressure method: air bubble growing at a tip of a capillary inside the bulk aqueous solution.

An equation which relates adsorption $\Gamma(t)$ to the diffusion $D$ of solute molecules from the bulk to the interface as well as to their back diffusion was first obtained by Ward and Tordai..$^{16}$ It accounts for the diffusion of monomers from the bulk to interface, and also for the back diffusion into the bulk as the interface becomes more crowded. It is widely used to analyze the adsorption process from the dynamic surface tension measurements. ${ }^{5}$ :

$\Gamma(t)=2 \sqrt{\frac{D}{\pi}}\left\{c_{b} \sqrt{t}-\int_{0}^{\sqrt{t}} c(\tau) d(\sqrt{t-\tau})\right\}$

where $c$ is the bulk surfactant concentration, $D$ the monomer diffusion coefficient, $c(\tau)$ the concentration in the subsurface, and $\tau$ is a dummy variable of integration. The solid lines in Figure $2 \mathrm{~b}$ are fits according to Equation 2, which has been solved numerically using the code developed by Li at al. ${ }^{17}$. It is assumed that the isotherm of surface tension at equilibrium can be described by semiempirical Szyszkowski Equation which is valid in semidilute regime:

$\gamma_{o}-\gamma=B \ln (1+a c)$

(Eq. 3)

Parameters $\mathbf{K}_{\mathrm{L}}(a)$ and $\boldsymbol{\Gamma}_{\max }(B)$ for linalool and benzyl alcohol have been obtained from the surface tension isotherms measured with bubble pressure tensiometer at 20 s surface age time. The approximation of the surface tension to the infinite-time limit $\gamma_{\mathrm{eq}}$ (Table $\mathrm{S}_{3}$, Supporting Information) has been done using method suggested by Christov et al. ${ }^{14}$ More details are given in the Supporting Information.

Dynamic surface tension of linalool solution with a concentration of $6,4 \cdot 10^{-4} \mathrm{~mol} / \mathrm{L}$ can be well fitted by WardTordai model (Figure $2 \mathrm{~b}$ ). The experimental value of $\gamma$ at $20 \mathrm{~s}$ surface-age time is slightly higher than that evaluated from Equation 3, while the estimated diffusion coefficients for linalool and for 1-decanol are an order of magnitude lower than that evaluated from Einstein relation (Table S2, Supporting Information). This result indicates an existence of the adsorption barrier for the molecules to transform from sub-interfacial layer to the interface.

The estimated equilibrium surface tension of the $\mathbf{1}^{-}$ decanol solution is considerably lower than that measured at $20 \mathrm{~s}$ surface age (Table S2). The experimental curve for the solution of benzyl alcohol can not be described in the frame of Ward-Tordai model, since the evaluated diffusion coefficient does not have a physical sense (Figure $\mathrm{S} 1$ ).

Data presented in Figure a,b convincingly demonstrate qualitative and quantitative difference in the behavior of selected alcohols both on a short-term scale and at (quasi)equilibrium conditions. Benzyl alcohol has a relatively good solubility in water (Table 1), so that its solutions exhibit practically a time-invariant surface tension in the whole studied concentration and time-scale ranges. The low interfacial activity of benzyl alcohol is due to small energetic gain in moving solvated molecules from solution to the interface. Poorly soluble 1-decanol reveals a 
high surface activity at a time scale of seconds, i.e. in a quasi-equilibrium adsorption state. Its retarded diffusivity is clearly seen in the isotherm at $40 \mathrm{~ms}$ time (Figure 2a) and in respected kinetic curve in Figure $2 b$.

The most interesting behavior from the practical point of view has been found for linalool, which shows a high dynamic activity already at a time scale of tens of milliseconds. Linalool exhibits a higher diffusivity in diluted solutions than that of 1-decanol and a higher dynamic and equilibrium interfacial activity as compared to benzyl alcohol, resulting in 100 times lower amount of linalool to produce the same interfacial effect as benzyl alcohol (Table $S_{2}$ ). We note that measurements of the surface tension of solutions of poorly soluble aroma molecules are complicated by so called mesoscale solubility of organic liquids in water. ${ }^{18}$ During tensiometric measurements, the mesoscale solubility in a form of "heterogeneous" oil droplets has to be taken into account. The mesoscale droplets, which serve as reservoirs and affect the diffusion-limited regime of adsorption, can be removed from the solution by filtration. According to our observations, the transition from molecular to mesoscale solubility of tested aroma molecules is detected at the concentrations below the solubility limit reported for these compounds in literature, that suggest a revision of the published solubility data.

Figure za compares surface tension isotherms of linalool and of conventional surfactant sodium dodecyl sulfate (SDS) in MQ water. As clearly seen, linalool exhibits a higher interfacial activity, especially in a millisecond range. In contrast to micelle-forming SDS solutions (Figure $2 \mathrm{~d}, \mathrm{~A}$ ), the cut-off in the concentration dependence of the surface tension of linalool solutions is attributed to the limit of its molecular solubility. The fact that the lowest surface tension is similar for both systems is rather accidental, since this value varies for the saturated solutions of aroma molecules. A practical conclusion for inkjet formulations is that a typically desired value of $\gamma$ of 55 $\mathrm{mN} / \mathrm{m}$ at $\sim 50 \mathrm{~ms}$ surface age time can be achieved with a three times lower amount of linalool as compared to that of a conventional surfactant (Figure 2a).

Obviously, important properties and functions of micelleforming surfactants are not innate for amphiphilic organic oils. Therefore, an intriguing question was whether the mixtures of conventional surfactants and of aroma molecules retain the specific features of individual solutions. Displayed in Figure $3 \mathrm{~b}$ are surface tension isotherms of SDS and linalool individual solutions, as well as of their mixtures with a constant fraction of linalool of 0,5 versus total molar concentration of surfactants in solution. (a)

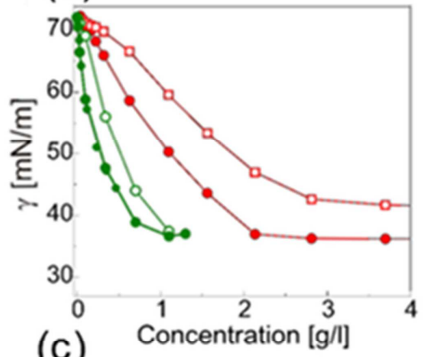

(b)

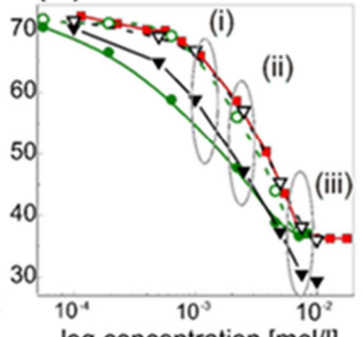

(c)

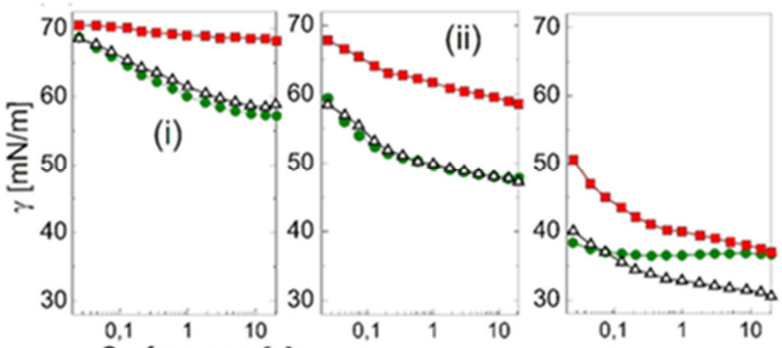

(d) Surface age [s] Surface age [s] Surface age [s]

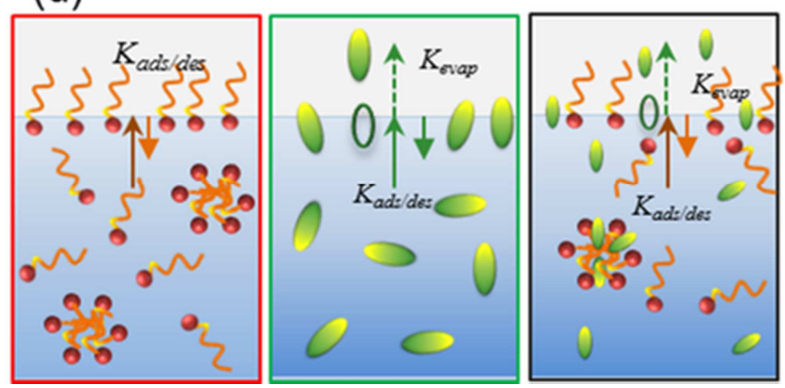

Figure 3 (a, b) Surface tension isotherms at $45 \mathrm{~ms}$ (open symbols) and at $20 \mathrm{~s}$ (filled symbols) versus concentration in MQ water of SDS (red squares), of linalool (green circles) and in (b) of mixed solutions of SDS and linalool (black triangles) with a constant mole fraction of linalool of 0,5 . All measurements have been done at $25^{\circ} \mathrm{C}$. (c) Surface tension versus surface age for the selected solutions of SDS (red squares), linalool (green circles) and their mixtures (black triangles) with a total surfactant(s) concentration i, ii and iii of o,ooo8, 0,02 and $0,1 \mathrm{~mol} / \mathrm{L}$, respectively. Sketches in (d) illustrate possible distribution of the volatile (linalool) and conventional (SDS) surfactants within the phases (water and air) and at their interface as a result of adsorption-desorptionevaporation processes.

Quantitative analysis of these data is beyond the scope of this publication. Here we emphasize several important observations. In the concentration range below the critical micelle concentration (CMC) of SDS, the dynamic behavior of the mixtures is very similar to that of linalool solutions of the same concentration (Figure $3 \mathrm{c}$ i,ii). This observation can be interpreted as an enhancement of the intrinsic diffusivity of SDS molecules by lowering the adsorption barrier in the presence of linalool.

Around CMC of SDS $(8,3 \mathrm{mmol} / \mathrm{L})$ a clear synergetic effect of the surfactants is seen in that the surface tension of the mixture at a time scale of above $\sim 100 \mathrm{~ms}$ is significantly lower than that of the individual solutions of each surfactant with the same concentration (Figure $3 \mathrm{c}$, iii). The synergetic adsorption behavior of mixtures of 
charged and non-ionic surfactants is typically attributed to the formation of a denser adsorption layer as a result of the reduced electrostatic repulsion of the charged headgroups. ${ }^{19}$ From a dynamic point of view, with increasing bulk surfactant concentration typically a drop of the evaluated diffusion coefficient in several orders of magnitude is observed. It is explained by thermodynamic factors and by a statistical factor, related to the availability of an empty site for a new molecule to enter a crowded interface. ${ }^{5}$ The latter factor presumably explains the enhanced effective diffusivity of SDS in mixed solutions from the bulk towards the interface as a result of the property of aroma molecules to desorb from the interface into the air phase thus reducing the backward diffusion of SDS surfactants (Figure 3d).

The volatile behavior of surfactants has been studied using pendant drop method, which has been utilized in two combinations: in a closed cuvette allowing for a saturated atmosphere, and in air (Figure 4a). In the latter case the solvent (water) evaporates during the measurements, and at the same time the unsaturated atmosphere affects the adsorption-desorption equilibrium of the volatile surfactant.

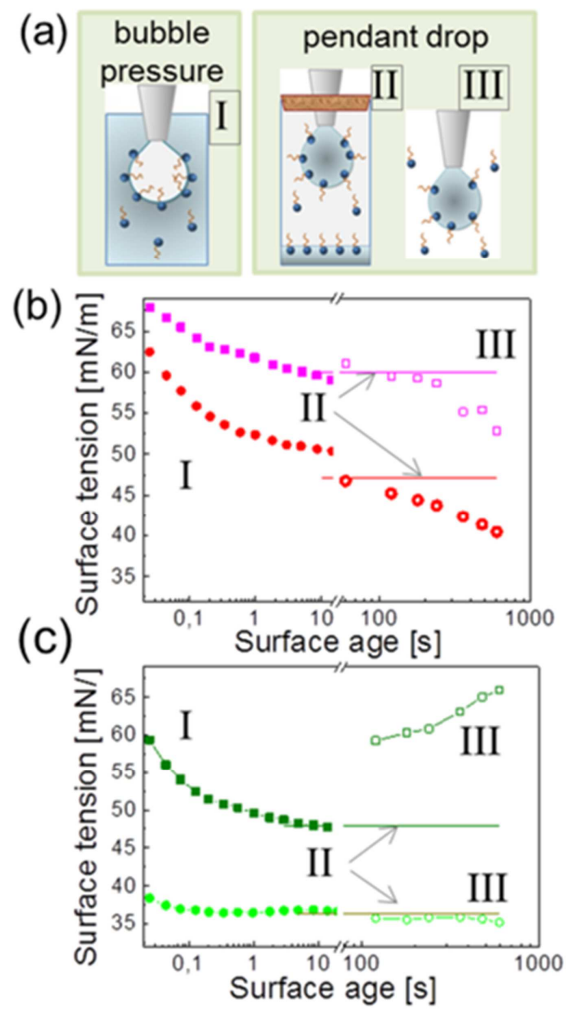

Figure 4 (a) Schematic representation of the experimental set-ups to measure surface tension: I - geometry of the maximum bubble pressure method, II and III - pendant drop method in a closed and open cuvette, respectively. (b) Surface tension of the SDS solutions with concentrations of $2.2 \cdot 10^{-3} \mathrm{~mol} / \mathrm{L}$ (red squares) and $5 \cdot 10^{-3} \mathrm{~mol} / \mathrm{L}$ (magenta squares) versus surface age measured by method I (filled symbols), by method III (open symbols) and by method II (solid lines). (c) Surface tension of linalool solutions with concentrations of $2,4 \mathrm{mmol} / \mathrm{L}$ (dark green circles) and 10 $\mathrm{mmol} / \mathrm{L}$ (green circles) versus surface age. Measurement methods as in (b).

Shown in Figure 4a,b are kinetic curves of the surface tension, collected over a large time scale by a combination of dynamic (I) and static (II) measurements. For both concentrations of SDS (above CMC) presented in Figure $4 \mathrm{~b}$ the equilibrium adsorption (constant surface tension value) is achieved on a time scale of tens of seconds. In the open system (III) on a longer time scale the surface tension continues to decrease as a result of the reduction of the drop volume (water evaporation) leading to an increased concentration of the surfactant in the pendant drop.

Different behavior is observed for linalool solutions (Figure 4c). While in the closed cuvette (system II) the equilibrium surface tensions measured by the pendant drop and by the bubble pressure methods are in a good agreement, in the open system the evaporation of the surfactant into the gas phase overcomes the effect of water evaporation, so that the surface tension of $2.4 \mathrm{mmol} / \mathrm{L}$ linalool solution increases with time and almost reaches that of water within 100 seconds. The constant surface tension of $10 \mathrm{mmol} / \mathrm{L}$ linalool solution is due to the compensation of the surfactant evaporation by the reduction of the drop volume as a result of water evaporation.

To clarify the effect of the volatile surfactants on the equilibrium wetting behavior and on the spreading kinetics of aqueous solutions we employed two kinds of measurements. In a first set a $\sim 40 \mu$ l-small pendant drop with a life time of minutes, i.e. with a preformed adsorbed layer, was brought into contact with a solid substrate (Figure 5a), and a dynamic wetting behavior (drop diameter and contact angle) has been monitored. Time dependencies of contact angle and of drop base diameter of drops of water and of $7 \mathrm{mmol} / \mathrm{L}$ solution of linalool on a freshly cleaned glass surface are presented in Figure $5 \mathrm{~b}$ in half-logarithm coordinates.

For both systems spreading process can be divided into two stages: fast spreading at $0-10 \mathrm{~ms}$ down to contact angle of $\sim 20-25^{\circ}$, and slow spreading up to $5^{\circ}$ in $1 \mathrm{~s}$. Figure 5 c shows time dependence of the squared drop base diameter for the fast spreading stage (at $\mathrm{t}<10 \mathrm{~ms}$ ) which can be presented as $d^{2}=k t$, where $\mathrm{k}$ is the spreading rate constant. Values of $\mathrm{k}$ determined from the experimental data for water and linalool solution are $470 \pm 20 \mathrm{~mm}^{2} / \mathrm{s}$ and $340 \pm 10 \mathrm{~mm}^{2} / \mathrm{s}$, respectively (Figure $4 \mathrm{c}$ ). The lower values of $k$ for linalool solution indicate that already on a time scale of $<10 \mathrm{~ms}$ linalool reduces the surface tension of water. Thus, with these experiments we assess the surface age times, which are even shorter than that currently accessible with bubble pressure tensiometry.

On the other side, the constant of the kinetic spreading regime is shown to be dependent on the surface tension: $:^{20}$

$k=\sqrt{\frac{\gamma R}{\rho}}$

where $\gamma$ is the surface tension, $R$ - radius of curvature, $\rho$ liquid density. The rate constants $k$ evaluated from Equation 4 result in $270 \mathrm{~mm}^{2} / \mathrm{s}$ and $220 \mathrm{~mm}^{2} / \mathrm{s}$ for water and 
linalool solution, correspondingly. The reduction of the spreading constant is presumably due to the fast decrease of the surface tension of water in the presence of linalool. The fact that the calculated from the experimental data and evaluated according to Equation 4 values of the spreading rate constant are of the same order of magnitude can be considered as a confirmation of the applicability of the spreading model for the studied systems.
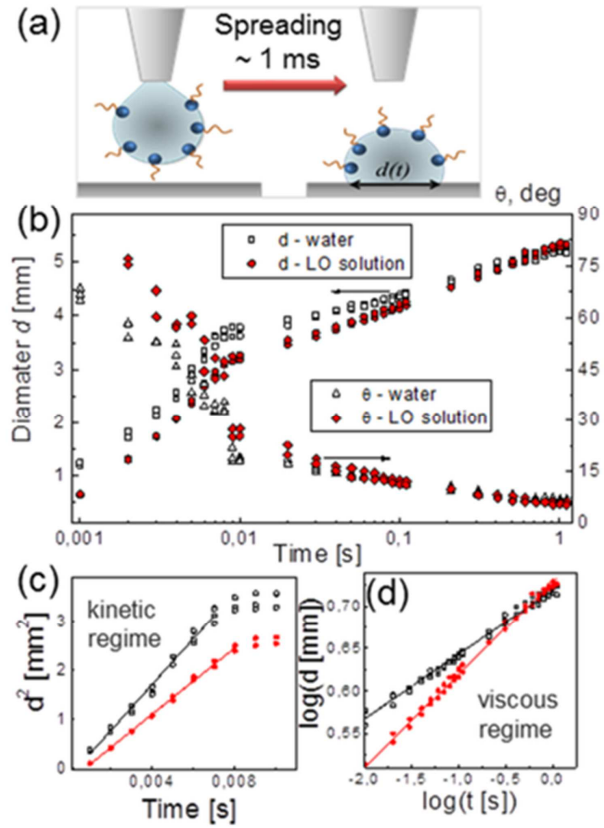

Figure 5 (a) Schematic of the experiments on wetting dynamics. (b) Drop base diameter (left hand axis - LHA) and contact angle (right hand axis - RHA) versus time for the spreading drops of water (black symbols) and drops of $0,375 \mathrm{~g} / \mathrm{L}$ solution of linalool in water (red symbols) on glass surface. (c,d) Analysis of the spreading kinetics of drops of water (black symbols) and of $0,375 \mathrm{~g} / \mathrm{L}$ solution on freshly cleaned glass surface in kinetic and in viscous regimes, correspondingly.

In a time range of $0,01-1 \mathrm{~s}$ a much slower spreading was observed with time exponents of 0,075 and 0,110 for water and for linalool solution, correspondingly (Figure 5d). These values are close to 0,1 , which is an indication of viscous spreading regime for the case of perfect spreading, as shown by Tanner ${ }^{21}$ and de Gennes..$^{22}$

$r(t)^{10}=A t$

(Eq. 5)

where $A=\frac{3 V^{3} \sigma}{\eta K}, \mathrm{~V}$ - drop volume, $\sigma$ - surface tension, $\eta$ liquid viscosity and $K$ - empirical coefficient close to 10 . Interestingly, in the viscous regime linalool accelerates spreading of water on glass, i.e. the effect is inversed as compared to the kinetic regime of spreading when the reduction of the spreading driving force resulted from the decreased surface tension of the linalool solution. This apparent contradiction can be attributed to the volatile behavior of linalool on a longer timescale: evaporation from the drop surface should cause depletion of linalool in the drop close to the triple-phase contact line. Consequently, a negative gradient of the surface tension appears from the wetting front to the middle of the droplet interface (Marangoni Effect), forcing the drop to spread faster. Thus, depending on the time scale of the process (as well as on the concentration of the added volatile surfactant) the volatile amphiphiles can either accelerate or retard the spreading behavior of aqueous formulations. These results may be relevant to the role of surfactants in governing interfacial particle motion. ${ }^{23}$

Model jetting experiments (Figure 6) demonstrate the appropriateness of volatile surfactants in controlling the contact line receding phenomena of liquid droplets under evaporation. Printed on polymer substrates pl-small droplets, containing conventional $\left(\mathrm{DX}_{4005} \mathrm{~N}\right)$ and volatile surfactants, have been analyzed with regards to their diameter (Figure S3b, Supporting Information), evaporation time (Figure $6 \mathrm{~b}$ ) and receding contact angle (Figure $6 c$ ).
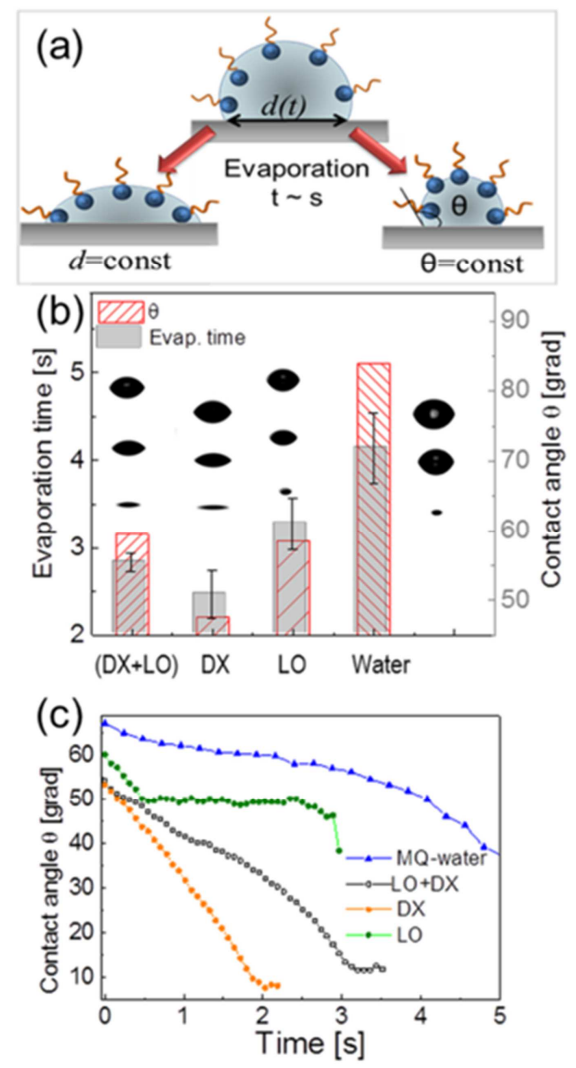

Figure (a) Schematic of the experiments on wetting dynamics and evaporation. (b) Estimated drop evaporation time (LHA) and contact angle at drop deposition (RHA) for the drops of water and of solutions containing $0,3 \%$ of $\mathrm{DX}_{400}{ }_{5} \mathrm{~N}$ (DX), o, $4 \%$ of linalool (LO), as well as of the mixture of $0,3 \%$ $\mathrm{DX}_{400} \mathrm{~N}$ and $0,5 \%$ linalool, as indicated. (c) Dynamics of receding contact angle of drops of solutions as in (b).

As clearly seen in Figure 6b, the smaller the primary contact angle the shorter is the droplet evaporation time. Droplets of fluoro-surfactant DX ${ }_{4005 \mathrm{~N}}$ wet the polymer surface well and evaporate in a pinned state keeping liquid/solid area constant during the whole evaporation time. Thus the reduction of droplet volume leads to a continuously declining contact angle (Figure 6c). Droplets of linalool solution transit within $0,5 \mathrm{~s}$ from "constant area" to "constant contact angle" mode. The mixture of both surfactants exhibits wetting behaviour which is a 
combination of both modes. Therefore, these experiments demonstrate that by mixing surfactants with different surface/interfacial activities, it is possible to tune the dynamic surface tension (Figure $\mathrm{S}_{4}$, Supporting Information) and surface modification properties of the formulations, in particular, to trigger the onset of the pinningdepinning transition of the contact line dynamics and thus the quality and resolution of printed structures. ${ }^{24,25}$ Furthermore, an improved functionality of the printed structures, e.g. conducting lines, is expected as a result of the reduced final concentration of the volatile amphiphile.

\section{Conclusions}

In summary, aroma molecules are shown to possess functional properties beyond the odor trigger, such as high dynamic activity and volatility. These findings are envisaged to launch multiple applications of these volatile amphiphiles, as well as to advance material science, as well as broad range of technologies ranging from surfaceemerging processes to medical diagnostics ${ }^{26,27}$ and to geosciences, e.g. to control the cloud droplet formation. ${ }^{28}$ Further work on quantification of the interfacial properties and classification of volatile surfactants from a reach class of aroma (fragrance) molecules is in progress.

Supporting Information. Details to materials section; results of the quantitative evaluation of the dynamic curves shown in Figure 2a; additional data set to the printing experiments in Figure 6.

\section{AUTHOR INFORMATION}

\section{Corresponding Author}

* L.A.T. E-mail: tsarkova@colloid.chem.msu.ru; tsarkova@dtnw.de

\section{Present Addresses}

${ }^{\%}$ J.V. present address: Research School of Chemistry, Australian National University, Canberra, ACT 2601, Australia

\section{Author Contributions}

All authors have given approval to the final version of the manuscript.

\section{Funding Sources}

The research has been done in the framework of the ERA.Net RusPlus (Project JetVA). The financial support by the Russian Foundation for Basic Research (RFBR) according to the research project № 18-53-76005 is acknowledged.

\section{REFERENCES}

1. Kraft, P.; Bajgrowicz, J. A.; Denis, C.; Fráter, G. Odds and Trends: Recent Developments in the Chemistry of Odorants. Angewandte Chemie International Edition 2000, 39 (17), 29803010 .

2. Firestein, S. How the olfactory system makes sense of scents. Nature 2001, 413 (6852), 211-218.

3. Brattoli, M.; de Gennaro, G.; de Pinto, V.; Loiotile, A. D.; Lovascio, S.; Penza, M. Odour Detection Methods: Olfactometry and Chemical Sensors. Sensors 2011, 11 (5), 5290-5322.

4. Berg, J. C., An introduction to interfaces and colloids: The bridge to nanoscience. 2009; p 1-785.
5. Eastoe, J.; Dalton, J. S. Dynamic surface tension and adsorption mechanisms of surfactants at the air-water interface. Advances in Colloid and Interface Science 2000, 85 (2), 103-144..

6. Dukhin, S. S.; Kretzschmar, G.; Miller, R., Dynamics of Adsorption at Liquid Interfaces Theory, Experiment, Application. Elsevier: Amsterdam, 1995.

7. Miller, R.; Aksenenko, E. V.; Fainerman, V. B. Dynamic interfacial tension of surfactant solutions. Advances in Colloid and Interface Science 2017, 247, 115-129.

8. Thiele, M. J.; Davari, M. D.; Hofmann, I.; König, M.; Lopez, C. G.; Vojcic, L.; Richtering, W.; Schwaneberg, U.; Tsarkova, L. A. Enzyme-Compatible Dynamic Nanoreactors from Electrostatically Bridged Like-Charged Surfactants and Polyelectrolytes. Angewandte Chemie International Edition 2018, 57 (30), 9402-9407 DOI: doi:10.1002/anie.201805021.

9. Roché, M.; Aytouna, M.; Bonn, D.; Kellay, H. Effect of Surface Tension Variations on the Pinch-Off Behavior of Small Fluid Drops in the Presence of Surfactants. Physical Review Letters 2009, 103 (26), 264501.

10. de Saint Vincent, M. R.; Petit, J.; Aytouna, M.; Delville, J. P.; Bonn, D.; Kellay, H. Dynamic interfacial tension effects in the rupture of liquid necks. Journal of Fluid Mechanics 2012, 692, 499-510.

11. Glawdel, T.; Ren, C. L. Droplet formation in microfluidic T-junction generators operating in the transitional regime. III. Dynamic surfactant effects. Physical Review E 2012, 86 (2), 026308 .

12. The Chemistry of Inkjet Inks. World Scientific: 2009.

13. Bradbury, R.; Penfold, J.; Thomas, R. K.; Tucker, I. M.; Petkov, J. T.; Jones, C. The impact of alkyl sulfate surfactant geometry and electrolyte on the co-adsorption of anionic surfactants with model perfumes at the air-solution interface. J Colloid Interface Sci 2013, 403, 84-90.

14. Christov, N. C.; Danov, K. D.; Kralchevsky, P. A.; Ananthapadmanabhan, K. P.; Lips, A. Maximum Bubble Pressure Method: Universal Surface Age and Transport Mechanisms in Surfactant Solutions. Langmuir 20o6, 22 (18), 7528-7542 DOI: 10.1021/lao61239h.

15. Rosen, M. J.; Kunjappu, J. T., Surfactants and Interfacial Phenomena. John Wiley \& Sons: New York, 2012.

16. Ward, A. F. H.; Tordai, L. Time-Dependence of Boundary Tensions of Solutions I. The Role of Diffusion in TimeEffects. The Journal of Chemical Physics 1946, 14 (7), 453-461.

17. Li, X.; Shaw, R.; Evans, G. M.; Stevenson, P. A simple numerical solution to the Ward-Tordai equation for the adsorption of non-ionic surfactants. Computers $\mathcal{E}$ Chemical Engineering 2010, 34 (2), 146-153.

18. Rak, D.; Sedlák, M. On the Mesoscale Solubility in Liquid Solutions and Mixtures. The Journal of Physical Chemistry $B$ 2019, 123 (6), 1365-1374.

19. Hua, X. Y.; Rosen, M. J. Synergism in binary mixtures of surfactants: I. Theoretical analysis. Journal of Colloid and Interface Science 1982, 90 (1), 212-219.

20. Biance, A.-L.; Clanet, C.; Quéré, D. First steps in the spreading of a liquid droplet. Physical Review E 2004, 69 (1), 016301.

21. Tanner, L. H. The spreading of silicone oil drops on horizontal surfaces. Journal of Physics D: Applied Physics 1979, 12 (9), 1473-1484.

22. de Gennes, P. G. Wetting: statics and dynamics. Reviews of Modern Physics 1985, 57 (3), 827-863.

23. Ghafouri, A.; Zhao, M.; Singler, T. J.; Yong, X.; Chiarot, P. R. Interfacial Targeting of Sessile Droplets Using Electrospray. Langmuir 2018, 34 (25), 7445-7454.

24. Kajiya*, T.; Kaneko, D.; Doi, M. Dynamical Visualization of "Coffee Stain Phenomenon" in Droplets of Polymer Solu- 
tion via Fluorescent Microscopy. Langmuir 2008, 24 (21), 1236912374 .

25. Tsarkova, L., Viktorova J., Yakushenko A., Offenhäusser. Forschungszentrum Lülich GmbH. WO2018DEoo151, 22.05.2018.

26. Zaitsev, S. Dynamic surface tension measurements as general approach to the analysis of animal blood plasma and serum. Advances in Colloid and Interface Science 2016, 235, 201213.

27. Wolf, S.; Jovancevic, N.; Gelis, L.; Pietsch, S.; Hatt, H.; Gerwert, K. Dynamical Binding Modes Determine Agonistic and Antagonistic Ligand Effects in the Prostate-Specific G-Protein Coupled Receptor (PSGR). Scientific Reports 2017, 7 (1), 16007.

28. Sareen, N.; Schwier, A. N.; Lathem, T. L.; Nenes, A.; McNeill, F. Surfactants from the gas phase may promote cloud droplet formation. PNAS 2013, 110 (8), 2723-2728. 
Table 1. Molecular and physico-chemical characteristics of aroma molecules presented in Figure 1 and $2 a$

\begin{tabular}{|l|l|l|l|l|l|l|}
\hline Compound & $\begin{array}{l}\text { Molecular } \\
\text { formula }\end{array}$ & Structural formula & $\begin{array}{l}\text { Mol. } \\
\text { weight, } \\
\text { g/mol }\end{array}$ & $\begin{array}{l}\text { Solubility } \\
\text { in water, } \\
\text { g/l }\end{array}$ & $\begin{array}{l}\text { Boiling } \\
\text { point, }{ }^{\circ} \mathrm{C}\end{array}$ & $\begin{array}{l}\text { LogP }_{\text {ow }}^{\text {[a] }} \\
\text { Geraniol }\end{array}$ \\
\hline $\mathrm{C}_{10} \mathrm{H}_{18} \mathrm{O}$
\end{tabular}

${ }^{[a]} \log \mathrm{P}_{\mathrm{ow}}$ - partition coefficient of an amphiphile in a mixture on n-octanol and water

\section{Table of Contents artwork}

Dynamic surface tension is an important parameter which defines processes and technologies dealing with newly creating interfaces, such as spraying, coating, ink-jet printing, microfluidics, cleaning. Aroma molecules are shown to possess functional properties beyond the odor trigger, such as high dynamic activity at ms time scale and volatility at a scale of seconds, Such dynamic volatile surfactants are envisaged to be of high value in material science and manufacturing.

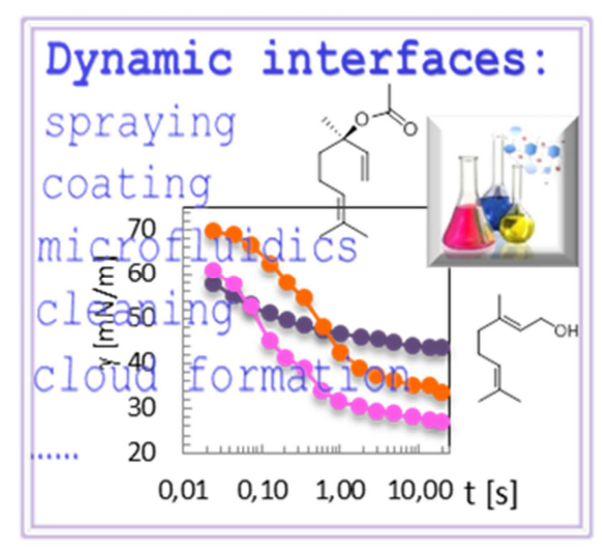




\section{Aroma molecules as dynamic volatile surfactants: functionality beyond the scent}

Oxana A. Soboleva, ${ }^{\#}$ Pavel V. Protsenko, ${ }^{\#}$ Vadim V. Korolev, ${ }^{\#}$ Jekaterina Viktorova, ${ }^{\dagger}$ Alexey Yakuschenko, ${ }^{\dagger}$ Ruth Kudla, ${ }^{\ddagger}$ Jochen S. Gutmann, ${ }^{\ddagger \&}$ and Larisa A. Tsarkova*\#‡

\# Chair of Colloid Chemistry, Faculty of Chemistry, Moscow State University 119991 Moscow, 1-3 Leninskiye Gory, Russia

${ }^{\dagger}$ Isitfresh GmbH, Technologiezentrum am Europaplatz, Dennewartstraße 25,52068 Aachen, Germany

${ }^{\ddagger}$ Deutsches Textilforschungszentrum Nord-West gGmbH (DTNW) Adlerstraße 1, 47798 Krefeld, Germany

${ }^{\&}$ Department of Chemistry \& CENIDE, University Duisburg-Essen, Universitätsstraße 5, 45141Essen, Germany

\section{Supporting Information}

\section{Materials and Methods:}

Solutions of conventional surfactants sodium dodecyl sulfat (SDS from Sigma-Aldrich), methylestersulfonat (MES from PT. Global Eco Chemicals Indonesia) anionic surfactants and DX4005N fluorosurfactant (Dynax Corporation) (Table S1) have been prepared by dilution of stock solutions. The curves presented in Figure 1b are exemplary measurements in course of titration experiments to obtain surface tension isotherm of respective surfactants.

Table S1. Conventional surfactants presented in Figure 1b

\begin{tabular}{|c|c|c|c|}
\hline Compound & $\begin{array}{c}\text { Molecular formu- } \\
\text { la }\end{array}$ & $\begin{array}{c}\mathbf{M}_{\mathbf{w}} \\
\text { g/mol }\end{array}$ & Application areas \\
\hline SDS & $\mathrm{NaC}_{12} \mathrm{H}_{25} \mathrm{SO}_{4}$ & 288,4 & In laundry and cosmetic formulations \\
\hline $\mathrm{MES}$ & $\begin{array}{c}\mathrm{C}_{16} \mathrm{H}_{33}\left(\mathrm{SO}_{3} \mathrm{Na}\right) \mathrm{CO} \\
\mathrm{OCH}_{3}\end{array}$ & 400 & In laundry formulations. Kraft point $\sim 27^{\circ} \mathrm{C}$ \\
\hline DX4005N & $\begin{array}{c}\text { Aqueous disper- } \\
\text { sion }\end{array}$ & - & $\begin{array}{c}\text { Provides rapid spreading, wetting and leveling } \\
\text { performance to various water-based coating } \\
\text { systems on low surface energy substrates }\end{array}$ \\
\hline
\end{tabular}

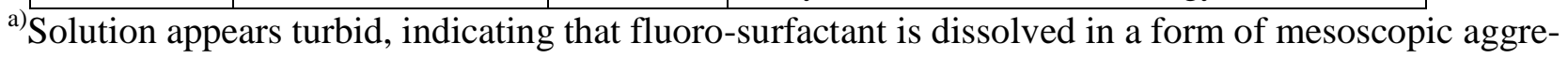
gates (confirmed by DLS measurements).

Evaluation of surface tension according to Ward-Tordai model:

Equation 2 has been solved numerically using the code developed by $\mathrm{Li}$ at al.[1]. It is assumed that the isotherm of surface tension at equilibrium can be described by semi-empirical Szyszkowski Equation 3 which is valid in semi-dilute regime.

Parameters $\mathbf{K}_{\mathbf{L}}(a)$ and $\boldsymbol{\Gamma}_{\max }(B)$ for linalool and benzyl alcohol have been obtained from the surface tension isotherms measured with bubble pressure tensiometer at $20 \mathrm{~s}$ surface age time. The approximation of the surface tension to the infinite-time limit $\gamma_{\mathrm{eq}}$ (Table S2) has been done using Equation S1:

$\gamma(t) \approx \frac{b_{\gamma}+\gamma_{e q} t_{a g e}^{1 / 2}}{a_{\gamma}+t_{\text {age }}^{1 / 2}}$

For 1-decanolsolutions parameters $B$ and $a$ of the Szyszkowski Equation have been evaluated from the data published in Ref. [2], were the static surface tension has been measured using ring tensiometer. 
Table S2. Results of the evaluation the kinetic curves in Figure 2b.

\begin{tabular}{|c|c|c|c|c|c|c|c|c|c|}
\hline & $\mathrm{C}, \mathrm{mol} / \mathrm{l}$ & $\gamma,(\mathrm{n}$ & /m) & $p C_{20}^{\text {a) }}$ & $\begin{array}{c}\mathbf{K}_{\mathrm{L}}, \\
\mathbf{m}^{3} / \mathbf{m o l}\end{array}$ & $\begin{array}{c}\Gamma_{\max }, \\
\mathrm{mol} / \mathrm{m}^{2}\end{array}$ & $\begin{array}{l}D_{,}^{b)} \\
\mathbf{m}^{2} / s\end{array}$ & $\mathrm{D},{ }^{\mathrm{c})} \mathrm{m}^{2} / \mathrm{s}$ & $\begin{array}{c}\gamma_{\mathrm{eq}}{ }^{\mathrm{d})} \\
\mathrm{mN} / \mathrm{m}\end{array}$ \\
\hline & & $\begin{array}{l}45 \\
\mathrm{~ms}\end{array}$ & $\begin{array}{c}20 \\
s\end{array}$ & & & & & & \\
\hline 1-decanol & $1.9 \times 10^{-4}$ & 70 & 58.7 & 3.7 & 39.54 & $6.4 \times 10^{-6}$ & $2.5 \cdot 10^{-11}$ & $5.1 * 10^{-10}$ & 39.5 \\
\hline Linalool & $7.5 \times 10^{-4}$ & 65 & 59.4 & 3.1 & 7.24 & $3.6 \times 10^{-6}$ & $6.310^{-11}$ & $5.2 * 10^{-10}$ & 57.4 \\
\hline $\begin{array}{l}\text { Benzyl } \\
\text { alcohol }\end{array}$ & $9.2 \times 10^{-2}$ & 59 & 57.4 & 1.0 & 0.02 & $6.7 \times 10^{-6}$ & - & $6.2 * 10^{-10}$ & 55.5 \\
\hline
\end{tabular}

${ }^{a)} p C_{20}=-\log C_{20}$-parameter used to assess the efficiency of the adsorption of surfactant molecules at the interface. It refers to the surfactant concentration at which the initial surface tension of the solution is reduced by $20 \mathrm{mN} / \mathrm{m}$. The larger the value of $p C_{20}$, the higher is the adsorption efficiency of the surfactant.

b) Calculated according to Equation S1 (Ward-Tordai)

${ }^{c)}$ Evaluated according Einstein relation $D=x^{2} / 2 t$, where $x$ is a diffusion length of a monomer for the time interval $t$.

d) Equilibrium surface tension according to Szyszkowski Equation (S2).

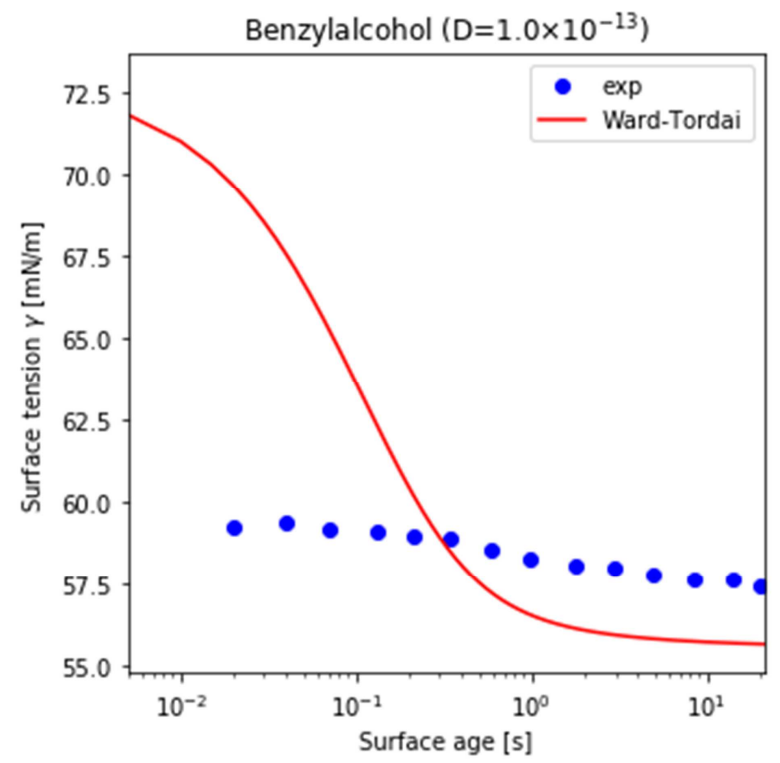

Figure S1. Surface tension versus surface age of $0,03 \mathrm{~g} / 1(0,00019 \mathrm{~mol} / \mathrm{l})$ solution of 1-decanol measured with bubble pressure tensiometer. Solid line is a fit according to Ward-Tordai model (Equation 2). 
(a)

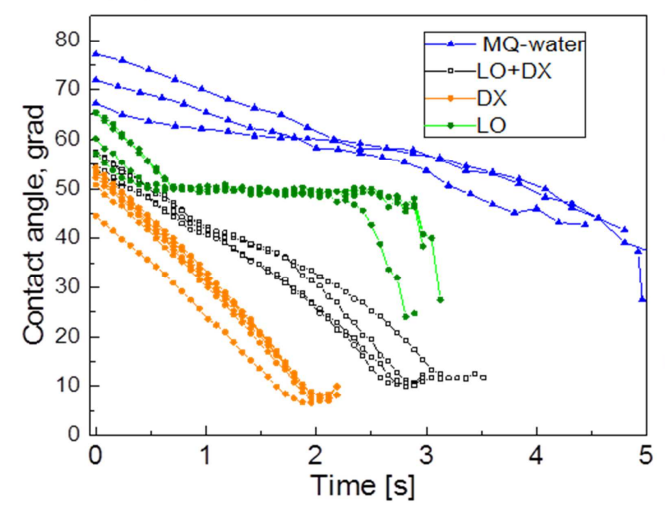

(b)

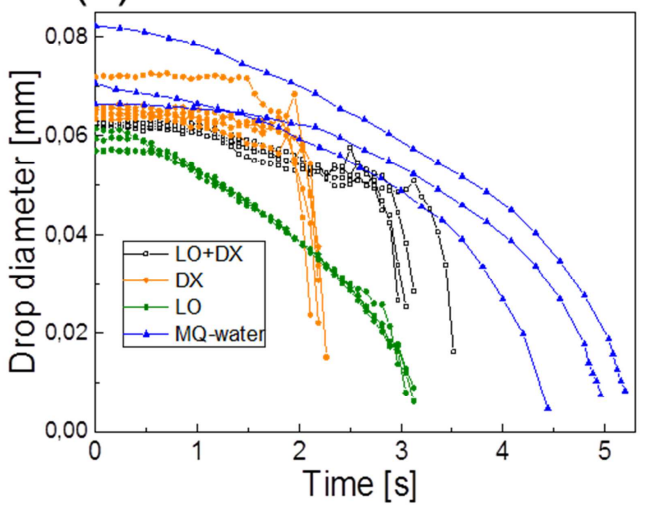

Figure S2. Droplet shape dynamics for MQ-water, 0,3\% solution of DX4005N (DX), 0,4\% solution of linalool (LO) and for the mixture of 0,3\% DX4005N and 0,5\% linalool: (a) dynamics of receding contact angle; (b) evolution of drop base diameter displayed for 4-5 measured droplets for each system.

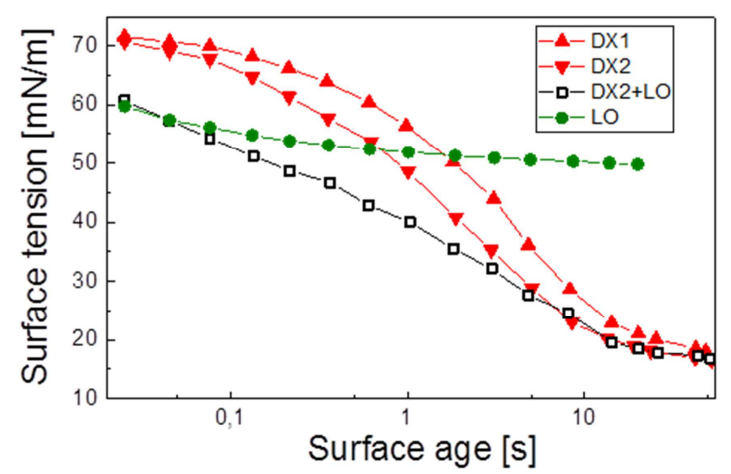

Figure S3. Kinetic curves of the surface tension of linalool and DX4005N solutions, as well as of their mixture. Details are summarized in Table S3.

Table S3. Details on the solutions and measurement curves presented in Figure S3

\begin{tabular}{|c|c|c|c|c|c|c|}
\hline \multirow{2}{*}{$\begin{array}{l}\text { Legend in } \\
\text { Figure S4 }\end{array}$} & \multirow{2}{*}{$\begin{array}{l}\text { Temperature } \\
\text { of the meas- } \\
\text { urement, }{ }^{\circ} \mathrm{C}\end{array}$} & \multicolumn{2}{|c|}{ Concentration } & \multicolumn{3}{|c|}{ Surface tension, $\mathrm{mN} / \mathrm{m}$} \\
\hline & & $\begin{array}{l}\text { DX4005N } \\
\mathrm{g} / 100 \mathrm{ml}\end{array}$ & $\begin{array}{l}\text { Linalool } \\
\text { g/ L }\end{array}$ & $\begin{array}{l}45 \mathrm{MS} \\
\text { (dynamic) }\end{array}$ & $\begin{array}{l}20 \text { s (qua- } \\
\text { si-static) }\end{array}$ & $\begin{array}{l}50 \mathrm{~s} \\
\text { (equilibrium) }\end{array}$ \\
\hline $\mathrm{LO}$ & 25 & & 0,17 & 69 & 60 & \\
\hline DX1 & 28 & 0,013 & - & 70,7 & 21,0 & 18,0 \\
\hline DX2 & 28 & 0,025 & - & 69,1 & 18,9 & 16,5 \\
\hline $\mathrm{DX} 2+\mathrm{LO}$ & 27 & 0,025 & 0,18 & 57,3 & 18,4 & 17,3 \\
\hline
\end{tabular}




\section{References:}

[1] Li, X., Shaw, R., Evans, G. M., \& Stevenson, P. (2010). A simple numerical solution to the WardTordai equation for the adsorption of non-ionic surfactants.Computers \& chemical engineering, 34(2), 146-153.

[2] Hommelen, J. R. (1959). The elimination of errors due to evaporation of the solute in the determination of surface tensions. Journal of Colloid Science, 14(4), 385-400. 